
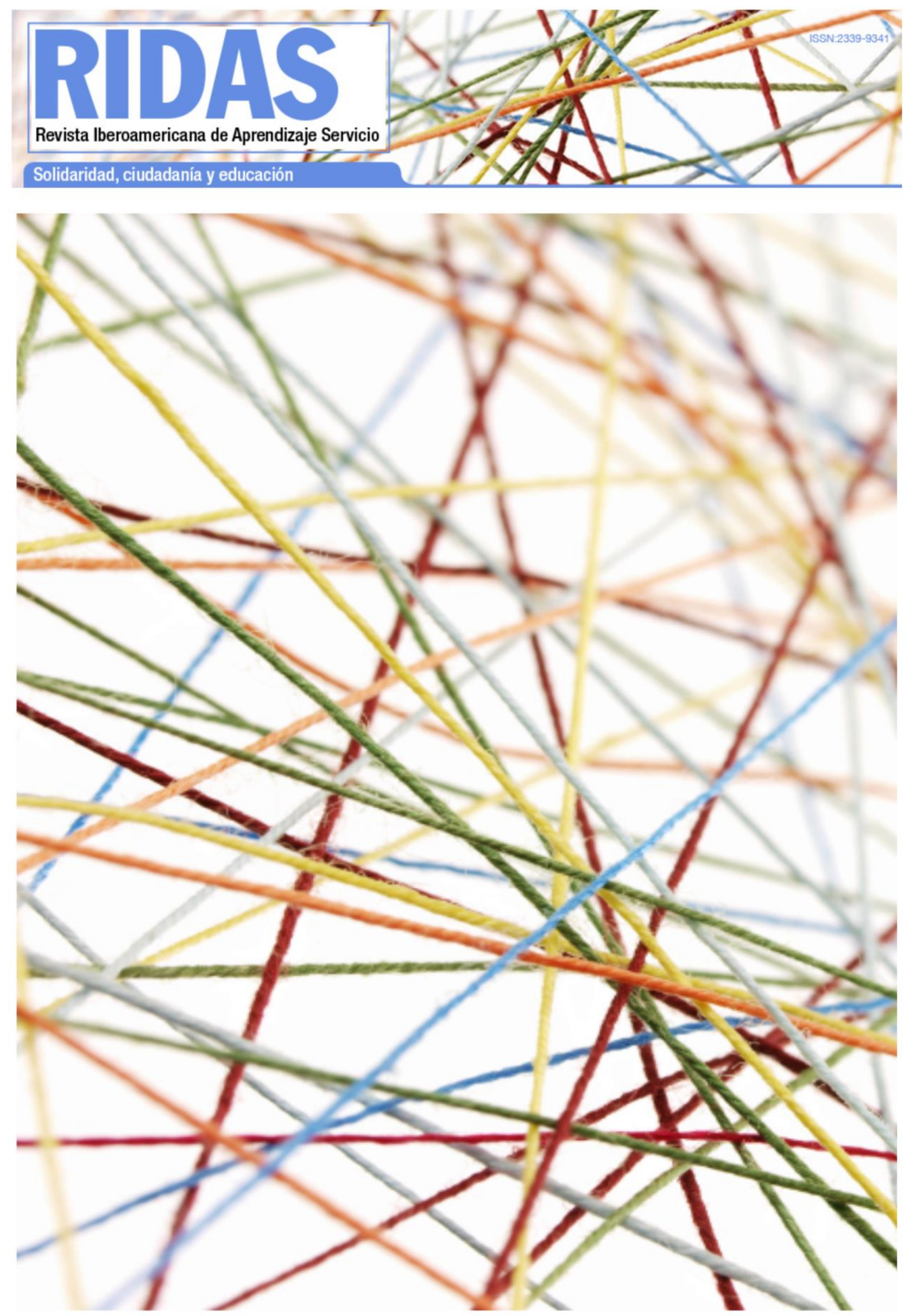


\title{
Aprendizaje-servicio y formación inicial docente. Factores que determinan el desarrollo de habilidades transversales
}

\author{
María Marques \\ Macarena Angulo \\ Luis Cáceres \\ Pontificia Universidad Católica de Chile, Chile
}

\section{Resumen}

El presente estudio indaga en el desarrollo de habilidades transversales a través de cursos que utilizan la metodología aprendizaje-servicio. Esta investigación cualitativa diseñó y validó una entrevista semiestructurada y un grupo focal, aplicados a 20 y 8 estudiantes de pedagogía respectivamente, los cuales fueron seleccionados por muestreo no probabilístico accidental. Para analizar la información recopilada se utilizó la codificación abierta. Los hallazgos muestran que los factores que inciden en el desarrollo de habilidades durante las experiencias de aprendizaje-servicio se relacionan con: los saberes previos de los estudiantes y la forma en que ellos los conectan con la experiencia; las oportunidades vinculadas con el tiempo de permanencia, las características del contexto y la mediación pedagógica; y por procesos que se viven durante la realización del servicio junto a los espacios de participación e involucramiento que la experiencia práctica debe comprender. Se discute y concluye en torno a cómo la presencia o ausencia de cualquiera de estos elementos afectará al desarrollo de habilidades durante la experiencia aprendizajeservicio.

\section{Palabras clave}

Aprendizaje-servicio, desarrollo de habilidades, formación inicial docente.

Fecha de recepción: 12/I/2021

Fecha de aceptación: 25/III/2021 


\title{
Service-learning and initial teacher training. Factors that determine the development of transversal skills
}

\begin{abstract}
Service-learning experiences bolster the acquisition of disciplinary knowledge and promote the development of transversal skills such as confidence, leadership, commitment and social justice. This study investigates the development of transversal skills through courses using the service-learning methodology. The qualitative research designed and validated a semi-structured interview and a focus group, involving 20 and 8 student teachers respectively, who were selected by accidental non-probabilistic sampling. Open coding was used to analyse the information collected. The findings show that the factors that bolster the development of skills during service-learning experiences are linked to the students' prior knowledge and the way in which they connect this knowledge to the experience; the opportunities presented to the students depending on the length of the experience, the characteristics of the context and the pedagogical support received, as well as the processes the students engaged with during the service, together with the spaces for participation and involvement that the practical experience included. The paper discusses and draw conclusions on how the presence or absence of any of these elements will affect the development of skills during the service-learning experience.
\end{abstract}

\section{Keywords}

Service-learning, skills development, initial teacher training. 


\section{Introducción}

Actualmente, una gran cantidad de programas de formación en educación terciaria se ven afectados por una importante brecha entre teoría y práctica (Colén y Castro, 2017) que, en el área educacional, se manifiesta en el choque con la realidad que sufren los docentes al iniciar el ejercicio profesional (Solís et al., 2016). En este contexto, las instituciones de educación superior tienen la tarea de brindar a sus futuros docentes instancias para el desarrollo de habilidades socioemocionales (Gutiérrez-Torres y Buitrago-Velandia, 2019), de pensamiento crítico y resolución de problemas (Gil, 2018) que les faciliten, en su desempeño profesional, sortear esta brecha. Con la intención de responder a tal objetivo, numerosos estudios han propuesto el aprendizajeservicio como una metodología que permite, a las instituciones de educación superior, disminuir la distancia entre "la experiencia práctica de servicio a la comunidad y la formación en conocimientos, habilidades y actitudes" (Batlle, 2013, p.21) a través de una acción andamiada por un importante componente reflexivo (Mayor y Rodríguez, 2016).

En ese sentido, este artículo presenta una investigación realizada en una universidad que implementó la metodología aprendizaje-servicio con el propósito de:

$$
\begin{aligned}
& \text { "[...] desarrollar una docencia } \\
& \text { que genere aprendizajes } \\
& \text { significativos basados en el }
\end{aligned}
$$

vínculo con la sociedad"; aportar al desarrollo de las comunidades por medio de los proyectos realizados por los estudiantes en el contexto de sus actividades académicas; y potenciar en los estudiantes el desarrollo del compromiso social y la formación en valores" (Jouannet et.al., 2013, p.201).

Bajo este marco, se presentan los hallazgos sobre las percepciones que tiene el estudiantado de las carreras de Pedagogía en Educación General Básica y Pedagogía en Educación Parvularia de la única sede regional de la Pontificia Universidad Católica de Chile (en adelante UC) respecto a las habilidades desarrolladas durante los cursos que han incorporado la metodología aprendizaje-servicio.

\section{Marco teórico}

\subsection{Sobre la metodología aprendizaje- servicio}

El aprendizaje-servicio es una metodología que emerge en 1903 en la Universidad de Cincinnati (Estados Unidos) como un movimiento de educación cooperativa que conecta trabajo, servicio y aprendizaje. Es John Dewey quien sienta los principios de la metodología, caracterizándola "como una enseñanza democrática, participativa e interactiva" (Arratia, 2008, p.62). Es en 1979 cuando Robert Sigmon define aprendizaje-servicio como un enfoque educativo experiencial cuya premisa es aprendizaje recíproco. Según Sigmon, el aprendizaje-servicio sólo ocurre cuando ambas partes se benefician de la experiencia, aquel que

Marques, M.; Angulo, M. y Cáceres, L. (2021). Aprendizaje-servicio y formación inicial docente. Factores que determinan el desarrollo de habilidades transversales. RIDAS, Revista Iberoamericana de Aprendizaje Servicio, 11, 1-22. DOI10.1344/RIDAS2021.11.1 
provee el servicio y aquel que lo recibe (Sigmon, 1979). Unos años más tarde, la National Society for Experiential Education, entidad que por años se enfocó en varios tipos de programas de educación experiencial, definió el aprendizaje-servicio como "cualquier experiencia de servicio cuidadosamente monitoreada en la que un estudiante tiene metas de aprendizaje intencionadas y reflexiona activamente respecto a lo que está aprendiendo a través de ella" (Furco, 1999, p.6).

Por utilizar los contenidos y las herramientas académicas en atención a necesidades genuinas de una comunidad (Furco y Billing, 2002), es posible afirmar que el aprendizajeservicio aumenta la adquisición de conocimientos disciplinares y promueve el desarrollo de habilidades transversales como la confianza, el compromiso social (Jenkins y Sheehey, 2011), el liderazgo y la justicia social (Groh et al., 2011).

\subsection{Habilidades transversales}

La evidencia muestra que a través de la metodología aprendizaje-servicio se incrementa el desarrollo de habilidades como el trabajo de equipo, la comunicación y el liderazgo, clara manifestación de desarrollo y maduración personal (Arratia, 2008); el desarrollo académico, como habilidades de pensamiento complejo y solución de problemas (Barrios et al., 2012); y otras habilidades y actitudes relacionadas con la participación cívica (Sandia y Montilva, 2020).

Particularmente, la bibliografía revisada sobre aprendizaje-servicio en el contexto de formación inicial docente (en adelante FID), muestra que, en una acción andamiada por un fuerte componente de reflexión (Mayor y Rodríguez, 2016; Páez y Puig, 2013), esta metodología constituye una buena herramienta para desarrollar la competencia social y ciudadana de los futuros docentes (Gil-Gómez et al., 2014; Santamaría-Goicuria y Martínez, 2018; Sotelino et al., 2019). En el mismo sentido, da cuenta de su idoneidad para discutir "las expectativas iniciales del pensar y del hacer abriendo nuevas sendas que cuestionan las posiciones hegemónicas del enseñar y el aprender" (Santamaría-Goicuria y Martínez, 2018, p.115).

Debido al éxito en su aplicación en especialidades como Derecho, Economía, Ingenierías, Ciencias Sociales o de la Educación, la metodología aprendizaje-servicio ha acaparado cada vez mayor atención en el ámbito de la Educación Superior (Zayas et al., 2019). De ahí que, por ejemplo, en España, al progresar la institucionalización de la metodología aprendizaje-servicio, aumentó la oferta de formación docente que incluye aprendizaje-servicio (Álvarez et al., 2017).

\subsection{Institucionalización de la metodología aprendizaje-servicio}

La institucionalización, según Curry (1992), es el final de un proceso en el que una innovación o programa queda completamente integrado a la estructura de la organización. Esta corresponde a la tercera de tres etapas que requiere un cambio institucional; a saber, movilización, implementación e 
institucionalización. Dicho proceso requiere de la constitución de una masa crítica de docentes que apoye y se comprometa genuinamente al interior de las facultades es primordial para que el aprendizaje-servicio se institucionalice en algún grado importante (Furco, 2001). De acuerdo con esta evidencia, la UC decide, en el año 2005, incluir el uso de la metodología aprendizaje-servicio como una de las metas estratégicas del Centro de Desarrollo Docente UC; unidad interna cuyo principal objetivo es promover una docencia de excelencia.

Para lograr esa meta, se desarrollaron capacitaciones y se asesoró a los docentes que trabajaron con aprendizaje-servicio en el proceso de planificación y diseño de sus cursos, facilitándoles estrategias para guiar las reflexiones y discusiones (Jouannet et al., 2012). Es así como el aprendizajeservicio se instaló como una alternativa marcada por su carácter curricular y pedagógico, elegida por un número importante de docentes y en diversas carreras de la universidad.

De este modo, como señalan Jouannet et al. (2012), la UC elabora, en el año 2011, un "modelo de institucionalización" basado en la rúbrica desarrollada por Andrew Furco (1999) y es la primera en América Latina que, explícitamente, institucionaliza la metodología aprendizaje-servicio en toda la universidad a nivel curricular de pregrado (Jouannet et al., 2015). De acuerdo con lo expuesto por estos autores, la Institucionalización del aprendizaje-servicio inicia con un proceso inductivo, a lo que sigue un diagnóstico y un piloto, para culminar con la implementación del aprendizajeservicio en la malla curricular de las carreras.

\subsection{Aprendizaje-servicio en la UC}

En un plano más específico, un curso que utiliza aprendizaje-servicio en la UC debe responder a tres aspectos: el académico, donde el estrecho contacto con la realidad posibilite mayores y mejores aprendizajes; uno relacionado con la calidad del servicio y con la contribución real para solucionar una problemática comunitaria; y finalmente con un aspecto que refiere a la formación valórica y actitudinal (Jouannet et al., 2013). En este sentido, los docentes que desarrollan cursos que incorporan la metodología deben intencionar experiencias que consideren el contexto de su clase, el servicio que van a brindar y las actividades de reflexión que acompañaran el proceso (Jouannet, 2013).

Dentro de los elementos que considera este modelo, tiene especial relevancia el desarrollo de habilidades transversales, especialmente beneficiadas por este tipo de experiencia (González et al., 2016) y de gran importancia en el ámbito académico y personal de los individuos.

En vista de los diversos beneficios que ha probado la utilización de la metodología aprendizaje-servicio, y siguiendo un riguroso protocolo (Caire et al., 2018), en el año 2016 se institucionaliza el modelo aprendizajeservicio en la única sede regional de la

Marques, M.; Angulo, M. y Cáceres, L. (2021). Aprendizaje-servicio y formación inicial docente. Factores que determinan el desarrollo de habilidades transversales. RIDAS, Revista Iberoamericana de Aprendizaje Servicio, 11, 1-22. DOI10.1344/RIDAS2021.11.1 
UC para potenciar en los estudiantes de Pedagogía en Educación Parvularia y Pedagogía en Educación General Básica el desarrollo de habilidades que les ayuden a desenvolverse mejor y a contribuir con la sociedad. A dos años de la instalación de la metodología aprendizaje-servicio en esta sede, se visualiza la necesidad de indagar en los factores que están incidiendo en que se desarrollen, o no, las habilidades que promueve el modelo aprendizajeservicio UC.

\section{Metodología}

\subsection{Método}

Este estudio de tipo cualitativo, por "tanto provisional y sometido a posibles cambios durante el transcurso de la investigación" (Quilaqueo y San Martín, 2008, p.157), busca lograr la comprensión integral del fenómeno en su contexto. Para profundizar en las percepciones de los estudiantes respecto a las habilidades que desarrollan durante los cursos que incorporan la metodología aprendizajeservicio, se recurre a la codificación. Este proceso asigna "un símbolo o marca indicativa a un segmento del cuerpo de registros, generalmente un fragmento textual" (Seid, 2016, p.7), en este caso de las entrevistas y del grupo focal.

\subsection{Participantes}

Para conformar el grupo de participantes se utilizó una técnica de muestreo no probabilístico accidental o consecutivo (Otzen y Manterola, 2017), modo de reclutamiento que permitió sumar casos hasta completar el número de sujetos deseado. El criterio de selección fue: I) haber cursado una o más asignaturas que hayan implementado aprendizaje-servicio, y II) haber firmado el consentimiento informado. En total, participaron veintiocho estudiantes de pedagogía; en detalle, catorce estudiantes de la carrera de Pedagogía en Educación Parvularia y catorce estudiantes de la carrera de Pedagogía en Educación General Básica.

\subsection{Instrumentos y materiales}

Para la realización de este estudio se utilizó una entrevista semiestructurada y un grupo focal para indagar en la realidad vivenciada por participantes. Estos instrumentos se diseñaron en función de tres dimensiones; a saber, procesos cognitivos, relacionales y educativos vividos en el contexto de sus experiencias aprendizaje-servicio.

Posteriormente, se procedió a validar el contenido de los instrumentos a través del juicio de seis expertos (Galicia et al., 2017), todos docentes con amplia trayectoria en metodología aprendizajeservicio. Los comentarios y observaciones fueron considerados en la versión aplicada a los participantes.

Previo a la aplicación de los dos instrumentos, se firmó un consentimiento informado. La entrevista se desarrolló presencialmente, en fecha y hora consensuada con los participantes. La aplicación de cada entrevista tomó aproximadamente cuarenta minutos y la del grupo focal cerca de una hora. Tanto el grupo focal como cada entrevista fueron registrados en audio para su posterior transcripción. 


\subsection{Procedimientos analíticos}

La información recolectada se transcribió y se analizó utilizando el software Atlas.ti 7.0. Se llevó a cabo una primera lectura de los datos que abrió la posibilidad de identificar, en los registros textuales, elementos a partir de los cuales se elaboraron códigos provisionales. Posterior a la primera lectura, las propuestas provisorias de códigos se afinaron en un proceso de sucesivas rectificaciones que emergieron de la comparación constante. Este proceso, denominado codificación abierta (Strauss y Corbin, 2002), supone examinar minuciosamente los registros. Al desmenuzar los textos se pudo acceder a los conceptos, ideas y sentidos contenidos en ellos (Quilaqueo y San Martín, 2008). En el proceso de codificación abierta, las categorías no estuvieron predefinidas, sino que emergieron de los mismos datos.

Luego de esta interpretación tentativa, y en la medida que progresó la codificación, la cantidad de conceptos creció. En un proceso iterativo de observación de los datos, los códigos que representaron los numerosos conceptos emergentes se compararon y dieron espacio a la generación de redes semánticas (proceso realizado en el software Atlas.ti). Dichas redes jugaron un rol fundamental en el proceso de análisis debido a que permitieron visualizar las posibles relaciones entre códigos, determinar su centralidad respecto al fenómeno analizado e identificar categorías que contaron con mayor nivel de abstracción y capacidad explicativa del fenómeno (Seid, 2016). Para cada categoría se identificaron propiedades que la explican y dimensiones que le otorgan mayor precisión a la descripción.

\section{Resultados}

A continuación, se presentan los conceptos, ideas y sentidos hallados en el discurso de los participantes sobre los factores influyentes en el desarrollo de habilidades durante la experiencia de aprendizaje-servicio. Los hallazgos se organizan en función de una categoría central denominada "Desarrollo de habilidades", la que involucra tres categorías conceptuales: "Saber personal", "Condiciones para el aprendizaje" y "Experiencia práctica".

\subsection{Categoría "Saber personal"}

Denominada "Saber personal", la primera categoría refiere al conocimiento previo de los estudiantes con relación a sí mismos y a la forma de relacionarse con los otros. En ese sentido, de esta categoría se desprenden dos propiedades: "Conocimiento intrapersonal", asociado con las preconcepciones y expectativas de los estudiantes sobre el aprendizaje; y la propiedad "Conocimiento interpersonal", relacionado con los vínculos establecidos durante la experiencia y con la comunicación, elemento clave para el desarrollo de habilidades relativas a la interacción con el entorno (Figura 1). 
Figura 1. Desarrollo personal

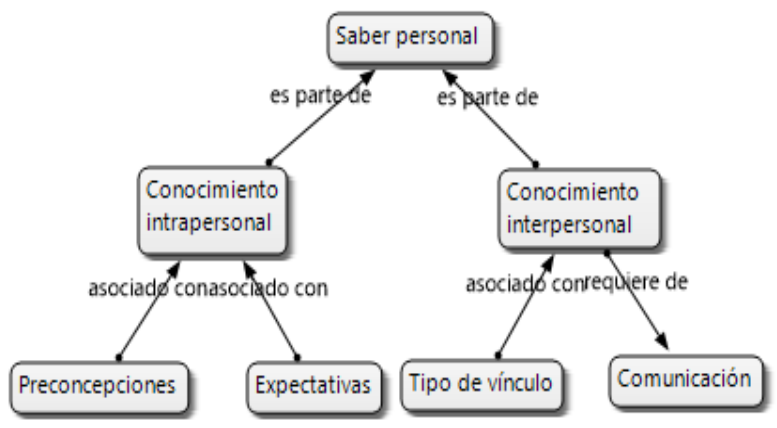

Fuente: elaboración propia

Los resultados del análisis de la propiedad "Conocimiento intrapersonal" muestran que los estudiantes manifiestan preconcepciones, entendidas como ideas formadas sin juicio crítico y sin tener en cuenta los datos de la experiencia. A este respecto, el concepto habilidad aparece recurrentemente vinculado a características como adquirible, desarrollable o innato. Como define una de las participantes de este estudio, "habilidades es como capacidades que uno puede tener o va desarrollando a través del tiempo o de diversas formas" (E1, Entrevista, 47:1-2). En relación con las expectativas, entendidas como la posibilidad razonable de que algo suceda, se observa cierta polarización en la forma en que los estudiantes expresan lo que creen, sucederá. Por un lado, algunos vinculan la experiencia aprendizaje-servicio con trascender, "dejar algo para alguien" (E5, Grupo focal, 190: 5-5) mientras otros, dicen tener dificultades para hacerse una idea sobre el aprendizaje-servicio:

- " "[...] no pensé nada y eso me faltaba porque no lograba entender lo que significaba, me costó mucho entender lo que era un aprendizaje-servicio. Me acuerdo que el profesor lo explicó muchas veces, estaba aún ahí en el proyecto, pero aun así no lograba encontrarle, eh, como dicen, ni pies ni cabeza" (E4, Grupo focal, 203:1-3).

Desde la percepción de los participantes, pareciera que las expectativas pueden construirse con cierto andamiaje. Un ejemplo de ello es este relato donde la participante pone en valor la introducción al curso:

- "[...] nos hablan mucho de las habilidades, nos hablaron mucho de los objetivos de este tipo de modalidad, que es aula multigrado, entonces uno ya va consciente de, más o menos, lo que quiere lograr [...] lo que hay que enfocarse [...] y se va autorregulando también [...] entonces yo creo que igual facilita bastante esa forma introductoria que tiene el programa para nuestras acciones futuras" (E3, Grupo focal, 244:26).

La propiedad "Conocimiento interpersonal" se relaciona con las habilidades que se requiere desarrollar para desenvolverse en un espacio social. Las dimensiones que permiten hacer una descripción de esta propiedad están asociadas con el "Tipo de vínculo" y la "Comunicación".

En cuanto a la dimensión "Tipo de 
vínculo", los datos permiten visualizar aquello que los estudiantes consideran necesario para establecer relaciones que posibilitan el desarrollo habilidades. En ese sentido, ellos hacen referencia a relaciones con pares, docentes guía y niños y niñas.

Respecto a los pares, se percibe la necesidad de establecer una relación profesional pero cercana a la vez. Un lazo convocado por el objetivo académico, pero en el cual se ven involucradas emociones relacionadas con la necesidad de ser validado por el grupo:

- "Siento que eso igual es súper importante en el trabajo en equipo, que yo sienta que lo que mi grupo está haciendo, es lo que yo también quiero que hagamos. Sentir que mi equipo me está escuchando, estoy participando, mis ideas también son consideradas" (E4, Grupo focal, 77:11-13).

En relación con el vínculo que establecen con el docente de aula, ellos perciben que para avanzar en su aprendizaje es:

- "[...] importante eso, esa palabra de apoyo en el camino [...] El hecho de dialogarlo también, de conversarlo, como más allá de, netamente, de rellenar informes [...] de repente uno, el estudiante siento yo, que necesitaba un apoyo [...] no sé si sicológico, pero tal vez sí porque de repente sí, se topa con cosas fuertes" (E16, Entrevista, 89:14-18).
Por último, los estudiantes perciben la fuerza que tiene el lazo que establecen o que pueden establecer con los niños y niñas:

- "Yo creo que toda esa organización que había, y aprovechar los tiempos que tuviéramos, nos sirvió para hacer, más allá de poder terminar con este proyecto [...] generar lazos, la cosa es cuando nosotros llegamos al colegio los niños ya nos veían y salían corriendo a encontrarnos [...] Entonces era ese lazo más allá de profesor alumno..." (E19, Entrevista, 89:1-6).

La dimensión "Comunicación", refiere a la acción de intercambiar información entre dos o más participantes. En este sentido, los estudiantes perciben que la comunicación entre pares resulta fundamental para el desarrollo de habilidades que los ayudan a mejorar como personas "en el plano personal [...] uno aprende a realizar trabajos en grupo, el trabajar en equipo... uno aprende a comunicarse, aprende a escuchar, aprende a llegar a acuerdos" (E8, Entrevista, 268:1-2). De la misma forma, la relación que establecen con el docente adquiere valor en la comunicación:

- " "...] la docente siempre tuvo la acogida para poder nosotros ir a consultar, siempre tuvo como redes abiertas por correo, su oficina, horarios que coordinamos [...] de repente va en uno poder expresarse hacia el docente..." (E1, Grupo focal, 282:1-3). 
El intercambio de información trasciende más allá del trabajo en equipo o del diálogo que se puede establecer entre docente y estudiante. Desde la percepción de los estudiantes, la comunicación que logran establecer con las familias les ayuda a promover el desarrollo de habilidades:

\section{- " "[...] tuvimos también que llevarle información a las} familias, involucrarnos con las familias y dar información [...] Entonces eso potenció concretamente lo que es servicio, la comunicación y todo [...] el trabajo que venía antes de eso" (E15, Entrevista, 60:3-5).

Así, la categoría "Saber personal" retrata la importancia del conocimiento que los estudiantes traen al aula, de los saberes intra e interpersonales que, de alguna forma, sientan las bases de la experiencia aprendizaje-servicio y el desarrollo de habilidades.

\subsection{Categoría "Condiciones para el aprendizaje"}

"Condiciones para el aprendizaje" se relaciona con aquellos elementos que potencian el desarrollo de habilidades en los estudiantes. De ella emergen dos propiedades, una relacionada con el tiempo y las características del contexto que se ha denominado "Oportunidades"; y otra llamada "Mediación pedagógica", que refiere al sentido otorgado al aprendizaje y a la reflexión como un elemento fundamental en la mediación (Figura 2).
Figura 2. Condiciones para el aprendizaje

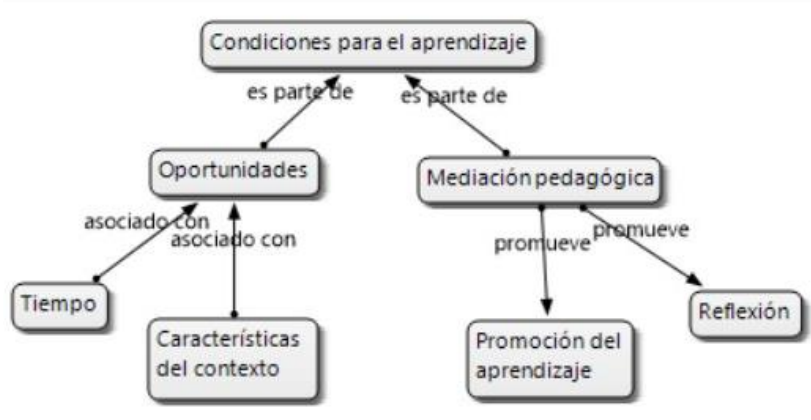

Fuente: elaboración propia

La primera propiedad muestra que durante la experiencia aprendizajeservicio deben existir ciertas condiciones que funcionan como "Oportunidades" para el desarrollo de habilidades. Esas oportunidades aparecen estrechamente ligadas a las dimensiones de "Tiempo", entendido como la duración de la experiencia y la permanencia en el contexto donde se realiza el aprendizaje-servicio; y a la dimensión "Características del contexto", referida a la apertura que la institución tuvo con los estudiantes y a su nivel de involucramiento con la experiencia.

Se puede observar que, de acuerdo con los estudiantes, el tiempo condiciona el vínculo que se desarrolla con los pares, así como con el equipo del centro. Señalan que, a menor tiempo de permanencia, disminuyen las oportunidades para desarrollar habilidades:

- " "[...] fue muy corto el tiempo, muy corto el periodo de aprendizaje y servicio, siento yo.

Marques, M.; Angulo, M. y Cáceres, L. (2021). Aprendizaje-servicio y formación inicial docente. Factores que determinan el desarrollo de habilidades transversales. RIDAS, Revista Iberoamericana de Aprendizaje Servicio, 11, 1-22. DOI10.1344/RIDAS2021.11.1 
Debería haber sido un poco más extenso para poder haber desarrollado más habilidades quizás, haber trabajado más con... tanto con el equipo mío de trabajo, tanto como con el jardín. $Y$ haber establecido un nexo más fuerte entre ambas partes" (E2, Entrevista, 91:1-4).

En cuanto a la dimensión "Características del contexto", los estudiantes perciben que, tanto el compromiso por parte de los otros actores como la autonomía que les brindan las instituciones, inciden en el desarrollo de habilidades. En relación con el compromiso por parte de los actores, una estudiante señala "en todos los centros donde estuvimos las personas estaban como muy dispuestas a ayudar a aprender [...] a tendernos la mano..." (E8, Entrevista, 174:2-4).

Así mismo, perciben ciertas restricciones, que de una u otra forma limitan el desarrollo de sus aprendizajes:

- "[...] la realidad que se vive en cada uno... es que uno es como muy estructurada; que no podemos hacer esto, que, como que imponían muchas cosas. Entonces nosotros teníamos que acomodarnos también a la realidad y al contexto que se estaba viviendo en el centro. $Y$ en el otro centro era como más abierto, pero igual como que los agentes que estaban ahí imponían muchas cosas" (E1, Grupo focal, 293:2-5).

La propiedad "Mediación pedagógica" se relaciona con el rol docente, específicamente con los procesos que este promueve para facilitar la enseñanza y el aprendizaje. Para "Mediación pedagógica" se identifican dos dimensiones, la "Promoción del aprendizaje" y la "Reflexión".

Promoción del aprendizaje refiere a los procesos a través de los cuales se facilita el desarrollo de habilidades, destrezas, conocimientos, conductas y valores. En esto el rol del profesor es fundamental y así lo perciben los estudiantes, "el recibir el proceso de retroalimentación, el acompañamiento que es súper importante $[\ldots]$ donde uno se siente perdido en ciertos aspectos" (E14, Entrevista, 85:2-4). Además de acompañar, ampliar el pensamiento es primordial, "si teníamos como una idea, al conversar con ella, se nos abrían muchas más ideas" (E1, Entrevista, 189:1-2). Es más, es posible observar que el aprendizaje está asociado con un trato más horizontal entre docenteestudiante: "más allá de un liderar de un profesor, fue un acompañamiento donde todos pudimos aprender" (E4, Entrevista, 127: 4-4).

Sin embargo, pareciera que la retroalimentación y el acompañamiento del profesor no logra romper la brecha entre el contenido y la práctica:

- "[...] porque cuando uno está haciendo las cosas en la misma práctica uno no está, porque... No está reflexionando profundamente acerca de lo que está haciendo, sabe uno que está haciendo algo importante, entonces en el momento de reflexionar uno como que 
recapitula para atrás y empieza a pensar y a ver y como a darle el sentido de lo que uno hace" (E13, Entrevista, 76:1-4).

Lo anterior se agudiza cuando se constata que los estudiantes perciben como importante el éxito o fracaso de sus implementaciones, sin lograr ver la estrecha relación que existe entre el contexto de la experiencia y el contenido que esperaban desarrollar:

- " "...] cada vez que íbamos al colegio [...] empezábamos... como ioh nos pasó esto! inos faltó esto! Después al ver los vídeos (teníamos que grabarnos) nos dimos cuenta como de varias cosas más... por el tema de las reflexiones escritas que teníamos que realizar, pero más que nada fue la conversación en grupo y el mismo trabajo en equipo, fue que pudimos observar si habíamos, digamos, cumplido el objetivo o si nos habíamos ido para otro lado" (E7, Entrevista, 57:2-6).

En cuanto a la dimensión "Reflexión", entendida como un acto cognitivo que implica pensar atenta y detenidamente sobre algo, los hallazgos muestran que se percibe como una parte importante de la mediación docente. Como menciona una estudiante: "Yo creo que ocupan mucha reflexión en el camino, [...] en el desarrollo, en el final y creo que es, que el trabajo de ustedes fue constante" (E12, Entrevista, 94:7-9). Y luego explica su importancia para el desarrollo de habilidades:

\section{- "[...] el proceso de reflexión es}

\section{Ridis.}

clave para cualquier proceso y la reflexión también recibimos retroalimentación, entonces todas las acciones que nosotros damos, que estaban reflejadas en las habilidades, fueron retroalimentadas tanto como por el profesor guía, con las personas que están en la sala de clases y con los compañeros. Porque hay cosas que uno no puede anotar cuando las está haciendo, pero otra persona cuando las ve si las rescata" (E12, Entrevista, 67:15).

En resumen, los hallazgos de la categoría "Condiciones para el aprendizaje" permiten ver que el desarrollo de habilidades durante la experiencia aprendizaje-servicio requiere oportunidades, tiempo suficiente para involucrarse con el contexto y participación por parte de la institución beneficiada; así como también requiere de mediación pedagógica que facilite instancias de reflexión y promueva la vinculación del contenido con la acción.

\subsection{Categoría "Experiencia práctica"}

La "Experiencia práctica" se relaciona con los procesos que se viven durante la realización del servicio, y con los espacios de participación e involucramiento que este debe comprender. En ese sentido, de esta categoría surgen dos propiedades llamadas "Aprender a aprender" y "Espacios de participación e involucramiento". La propiedad "Aprender a aprender" refiere a los procesos de pensamiento que acompañan la acción de los 
estudiantes; mientras que la propiedad "Espacios de participación e involucramiento" se desarrolla en torno al trabajo en equipo, la relación que establecen los estudiantes con el docente guía y la participación e involucramiento que logra el estudiante con la realidad educativa (Figura 3 ).

Figura 3. Experiencia práctica

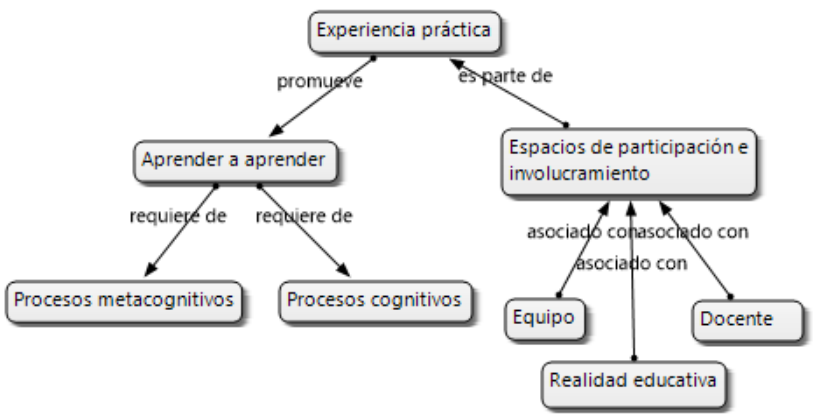

Fuente: elaboración propia

En cuanto a la Propiedad "Aprender a aprender" podemos organizar lo hallado en dos dimensiones: "Procesos cognitivos", referidos al pensamiento; y "Procesos Metacognitivos", que tienen que ver con la evaluación del mismo pensamiento. Los "Procesos cognitivos" refieren al conocimiento que tiene el individuo y cómo este determina la actividad que va a realizar; implica pensar sobre sí mismo, sobre la tarea y sobre la estrategia. Así lo retrata la siguiente intervención, donde se observan procesos cognitivos en torno a la estrategia:

- "[...] nos dimos cuenta de que cada vez, cuando hablamos más despacio, los niños tomaban más atención que cuando usábamos el mismo tono de voz $[\ldots]$ entonces es algo que he seguido replicando y he buscado nuevamente porque les llama mucho la atención y siento que sirve más..." (E4, Entrevista, 67:1-5).

Del mismo modo, la evidencia muestra que los procesos cognitivos que le permiten a los estudiantes enfrentarse a la acción, involucran la tarea y la estrategia "[...] queríamos hacer esto, queríamos hacer lo otro, y al final había tantas ideas que tuvimos que decir ipara!, yo localizo esto, tú esto otro..." (E3, Entrevista, 51:3-4); así como también implica conocimiento sobre sí mismo:

\section{- " "...] yo fui tomando mis} experiencias desde el principio del proceso y viviendo cómo evolucionaban, y cómo evolucionaba yo como persona [...] con relación a todo lo que yo hacía mal o bien, y ahí viendo yo también mis fortalezas como también mis debilidades..." (E2, Entrevista, 47:1-3).

La dimensión "Procesos metacognitivos" se asocia con la evaluación de los procesos de pensamiento. Estos dicen relación con el control o autorregulación que el sujeto que aprende tiene sobre los procesos de planificación, monitoreo y evaluación de la acción.

En relación con la planificación, una estudiante expresa que para poder enfrentar la experiencia "uno tiene que considerar integrar todo lo que uno aprende en la universidad, aprende en la vida y también dentro de uno" (E3, 
Entrevista, 91:4-5), proceso que otra estudiante profundiza al señalar que es necesario pensar en "qué estrategias íbamos a utilizar, qué herramientas, qué recurso y cuál iba hacer como nuestro objetivo de aprendizaje" (E7, Entrevista, 74:1-2).

Con respecto al monitoreo del pensamiento, encontramos evidencia sobre el seguimiento que una estudiante realiza de su proceso "había etapas como de mejora, donde nosotras mismas dando nuestra opinión, íbamos mejorando. Tomábamos en cuenta los puntos de vista de las tres para ir arreglando nuestro trabajo" (E4, Entrevista, 74:2$3)$.

$Y$ finalmente se aprecian procesos vinculados a la evaluación en tanto que otra participante expresa lo siguiente:

- "[...] analizar lo que uno ya ha hecho en el aula o con el equipo de trabajo [...] de repente uno actúa como robot en ciertas cosas porque tiene que ir rindiendo en algunas cosas [...] al socializarlo con otro, yo creo que recién ahí uno se hace consciente de todo el avance que ha tenido en el trabajo" (E8, Entrevista, 71:3-7).

Finalmente, la propiedad denominada "Espacios de participación e involucramiento", hace referencia a las habilidades necesarias para intervenir en una actividad e involucrarse con ella. Esta propiedad se vincula con las dimensiones "Equipo", "Docente" y "Realidad educativa". El "Equipo", tiene que ver con el trabajo con los pares durante la experiencia; el "Docente", se vincula con la colaboración del profesor guía durante el proceso; y la "Realidad educativa", refiere al involucramiento que logra el estudiante con el contexto.

En primer lugar, cabe mencionar que el trabajo en equipo emerge como un aspecto fundamental en la dimensión "Espacios de participación e involucramiento", siendo aparentemente el vínculo que, desde la percepción de los estudiantes, favorece el desarrollo de un conjunto de otras habilidades como comunicación, empatía y liderazgo:

- "Trabajo en equipo eh... Se complementa mucho con el liderazgo y tiene que ver con cómo, además de agregar el componente de... De aprovechar nuestras, nuestras virtudes, además tiene que ver con el componente de... De ser empático respecto de la forma de trabajo" (E20, Entrevista, 6:1-3).

Sin embargo, uno de los aspectos que sienten que puede afectar el potencial del trabajo en equipo, es conformar grupo con personas que no son cercanas, ya que se presenta repetidamente en el discurso de los estudiantes como un problema:

- " "...] bueno los grupos esa vez fueron al azar, yo por coincidencia quedé con la [...], que es mi amiga, entonces en ese sentido no hubo problemas digamos [...] pero había otra compañera que nosotras no conocíamos mucho y nos fuimos como enterando de sus, 
digamos, fortalezas y de sus debilidades durante el camino entonces fue bastante eh... no sé si complicado..." (E7, Entrevista, 218:1-8).

Respecto al vínculo con el docente guía, la evidencia muestra que se desarrolla un mayor involucramiento entre estudiante y docente cuando se trata de cursos de práctica que incluyen la metodología aprendizaje-servicio. En ese sentido, una estudiante responde a:

- "[...] en el primer aprendizajeservicio pudimos conocer antes el contexto al que íbamos, pudimos conversar antes con la profesora $y$ ver a los niños. Y eso después se nos hizo más ameno porque ellos quizás nos recordaban, sabían a qué íbamos a ir y la tía estaba como de acuerdo y llevaba el proceso con nosotros" (E12, Entrevista, 91:1-4).

Por el contrario, según la percepción de los estudiantes, este involucramiento es menor cuando se trata de otros cursos aprendizaje-servicio (teóricos o didácticos):

- "[...] bueno, con el equipo que había en el colegio nuestra relación no era tan... era más entre nosotras. Porque cuando nosotras íbamos la educadora hacía otras cosas, y la técnica que estaba ahí era como observación. Entonces el trabajo era como entre nosotras" (E4, Entrevista, 110:1-3).

En la dimensión "Realidad educativa", nos referimos al involucramiento que el estudiante logra con el contexto del servicio. En ese sentido, la necesidad real emerge como un elemento que invita a involucrarse; acción a menudo vinculada con el concepto de compromiso social:

- " [...] nosotros, para poder ofrecer un servicio, antes detectamos una necesidad [...] y cuando detectamos la necesidad ya no lo vimos como algo, como una nota o un juego; sino que hubo un compromiso de por sí, también con la educadora. De apoyarla en todo lo que ella necesitara porque así también íbamos aprender nosotras a trabajar..." (E10, Entrevista, 103:1-5).

Esta categoría señala lo expresado por los participantes en relación con los procesos del pensamiento que llevaron a cabo; además muestra características de la experiencia práctica en torno al trabajo con los pares, al involucramiento por parte del estudiante con el docente guía y la con realidad educativa.

\section{Discusión y conclusiones}

Las categorías en que se organizan los hallazgos de este estudio se articulan en una premisa general; la idea de que desarrollar habilidades es posible en la medida que, existe un conocimiento personal que facilita el logro del aprendizaje cuya consolidación requiere de ciertas condiciones que, en una situación de práctica, den espacio para participar, involucrarse y aprender a aprender. En consideración de lo importantes que son para el desempeño de una buena labor profesional, resulta 
pertinente destacar algunos elementos identificados como influyentes en el desarrollo de habilidades transversales.

En primer lugar, resalta la importancia que tienen los conocimientos previos de los estudiantes. En este sentido, las preconcepciones de habilidad que sostienen algunos de ellos son un tanto imprecisas. Ellos definen habilidad como algo con lo que se nace, se puede adquirir o desarrollar, conceptualización que al ser poco clara, puede formar una estructura interna que funcione como barrera para el desarrollo del propio conocimiento. Por ello, es importante que los estudiantes, con la ayuda del docente, sepan que, aunque trabajar una habilidad relacionada con las emociones es menos sencillo que aprender una actividad mecánica, todas se pueden desarrollar y perfeccionar (Baquero y Cárdenas, 2019). La metodología aprendizaje-servicio ha mostrado que la discusión y la guía que los docentes prestan en la ejecución de las actividades toma protagonismo durante este tipo de experiencias (Berríos et al., 2012). En este sentido, la literatura señala que, el éxito de una propuesta de aprendizaje-servicio requiere que el profesor incluya en el proceso elementos vinculados con la historia y las vivencias de los estudiantes (individuales y grupales); facilitándoles de este modo construir una base que les permita generar expectativas y avanzar hacia nuevos aprendizajes (Arratia, 2008). Lo anterior es reafirmado por Jouannet (2013), quién destaca la importancia del conocimiento que el docente tiene del contexto de su clase, especialmente en lo que dice relación con los saberes previos de sus estudiantes. Los hallazgos de este estudio muestran que, desde la percepción de los estudiantes, los profesores intentan dar contexto a la experiencia, muchas veces sin lograr conectar el aprendizaje-servicio con sus historias y vivencias previas, lo que se traduce en falta de comprensión de lo que están experimentando. En consideración de que el aprendizaje-servicio mejora la relación docente-estudiante (Jouannet et al., 2012; Jouannet et al., 2013), resulta conveniente que el docente apunte a conocerlos en profundidad para que, desde ese conocimiento, sientan respetada su individualidad y se logre instalar diálogos que gatillen el aprender a desaprender, dando un giro a las concepciones tradicionales y desarrollando la capacidad de transformación constante (Salum, 2018). El conocer a sus estudiantes permite, por tanto, que el profesor instale una discusión que, con la ayuda de los otros (pares y miembros de la comunidad donde prestan el servicio), derrumbe, como señalan SantamaríaGoicuria y Martínez (2018), aquellas conceptualizaciones ambiguas y limitantes, para construir otras más claras y empoderadoras a partir de información precisa.

En segundo lugar, interesa relevar el rol de las oportunidades que se le deben brindar al estudiante. A pesar de que evaluaciones realizadas al final de la experiencia aprendizaje-servicio UC muestran valoraciones positivas por parte de los representantes de las organizaciones socias respecto al apoyo experto de la universidad (Jouannet et al., 2013), los relatos analizados 
dejaron ver que el nivel de involucramiento de la institución en ocasiones se manifestó en acciones restrictivas para con los estudiantes o simplemente estaba ausente. De modo que, por falta de autonomía o retroalimentación correspondientemente, los estudiantes perciben que se coartan las oportunidades para desarrollar habilidades como el liderazgo y la resolución de problemas. Estas restricciones podrían atribuirse a dos factores: la percepción del socio comunitario en cuanto a la atención o no de una sentida necesidad (Pizarro y Hasbún, 2019) o al tiempo de la experiencia. En concordancia a que se ha reportado que "obtienen mejores resultados las intervenciones más duraderas" (Menor et al., 2017, p.81), el discurso de los estudiantes destaca el tiempo como un elemento que influye en que la experiencia aprendizajeservicio sea una oportunidad de desarrollo de habilidades. En este sentido, la percepción de los estudiantes es coherente con lo que postulan Pizarro y Hasbún (2019), que señalan la relación entre el nivel de vinculación con el medio y el tiempo de permanencia. En vista de esto, estos autores proponen que la universidad revise las opciones de vinculación y continuidad de los proyectos, dado que la cobertura que los cursos pueden ofrecer resulta insuficiente. Aparte del socio-comunitario y el tiempo, la retroalimentación y el acompañamiento que lleva el docente se relevan entre las condiciones que posibilitan el desarrollo de habilidades. Aunque se perciben esfuerzos por trabajar la habilidad reflexiva a través de ejercicios intencionados, son aparentemente insuficientes ya que la brecha entre teoría y práctica no se logra disminuir. Continuamos observando acciones, por parte de los estudiantes que prestan el servicio, que ellos perciben como importantes pero desconectadas del contenido que se discute en el aula de la universidad. Y nos preguntamos, ¿por qué los estudiantes siguen enfocándose en el éxito o fracaso de la implementación? ¿Cómo adquiere sentido el contenido en la práctica? ¿Cómo lo trabajo como docente? Corresponde a nosotros, los profesores, crear las oportunidades para que los estudiantes desarrollen habilidades como el pensamiento crítico, la resolución de problemas, la comunicación, el liderazgo y el compromiso social; situándonos como facilitadores o mediadores que verifican constantemente la existencia de una articulación entre lo teórico y lo práctico.

En tercer lugar, es imprescindible señalar la importancia de que la experiencia aprendizaje-servicio sea vivida por parte del estudiante de una forma muy consciente. Una experiencia que permita, entre pares y con otros actores, entrar en una discusión sobre los supuestos de enseñar y aprender que ellos traen consigo (SantamaríaGoicuria y Martínez, 2018), vivida con tal profundidad, que promueva una genuina conexión entre el individuo y el entorno. Solo a partir del hacer conscientes los procesos metacognitivos es posible pensar los fenómenos con una amplia perspectiva, y bajo esta premisa se sostiene la idea del "aprender a aprender" como un 
elemento decisivo para el éxito en el desarrollo de habilidades durante la experiencia aprendizaje-servicio. Si bien, en los resultados emergen referencias a procesos metacognitivos, los estudiantes los reportan como procesos más bien espontáneos, lo que deja entrever que actúan sin tener claridad del por qué. Pero ¿de dónde viene este hábito? Y, ¿cómo modificarlo? Podemos suponer que la escuela chilena, hace poco abierta a paradigmas de educación constructivistas (Caiceo, 2012), está promoviendo la acción mecanizada donde la reflexión no tiene lugar. Por ello, llevar a cabo un andamiaje que gatille este proceso representa una herramienta de gran potencial para trabajar las habilidades metacognitivas. Los estudiantes requieren un docente que promueva la habilidad reflexiva en una acción con sentido, centrándose en los procesos de diseño que estos llevan a cabo; guiándolos e invitándolos todo el tiempo a volver a la teoría, a dialogar con ella en el antes, durante y después de sus experiencias. Así como Schön (1998) indica en su propuesta de epistemología de la práctica (mejor conocida como Practicum): conocimiento en la acción, reflexión en la acción y reflexión sobre la acción y sobre la reflexión en la acción. A partir de lo dicho, se postula que este aprender a enseñar requiere de aprender a aprender, y que vivir la experiencia aprendizaje-servicio consciente es un factor determinante en el desarrollo de habilidades. En efecto, García y Cotrina (2015) señalan que sumado al prácticum y aplicado a la formación docente, el aprendizajeservicio otorga un perfil profesional de gran valor al estudiantado como resultado de su activa participación en propuestas y proyectos. Por ende, que los estudiantes se familiaricen con el ejercicio de evaluar sus procesos cognitivos representa una gran contribución a que la experiencia aprendizaje-servicio resulte exitosa en cuanto al desarrollo de habilidades.

Para concluir, resulta importante recalcar que, si cualquiera de estos tres factores está débil o ausente, afectará al desarrollo de habilidades. Dígase, el saber personal en la medida que el docente lo utiliza como punto de partida; las condiciones de aprendizaje dadas por las oportunidades que ofrece el contexto, en el terreno de práctica y la universidad; y la experiencia práctica que vive el estudiantado vinculada al aprender a aprender y a los espacios de participación e involucramiento. Por lo tanto, los hallazgos del presente estudio, aun cuando son acotados y se requiere ampliar el campo investigativo, sugieren que, si se tienen en consideración los factores anteriormente expuestos, la metodología aprendizaje-servicio constituye una oportunidad para mejorar el ejercicio docente y posibilita el crecimiento personal y profesional de todos los estudiantes.

\section{Referencias bibliográficas}

Álvarez, J., Martínez, M., González, H., y Buenestado, M. (2017). El aprendizaje-servicio en la formación del profesorado de las universidades españolas. Revista Española de Pedagogía, 75(267), 199-217. doi:10.22550/REP75-2-2017-02 
Arratia, A. (2008). Ética, solidaridad y "aprendizaje servicio" en la educación superior. Acta Bioethica, 24(1), 61-7.

Baquero, D., y Cárdenas, S. (2019). Habilidades transversales, desarrollo personal y profesional en la docencia a través de la plataforma Habilitic.

Conrado, 15(70), 421-428. Recuperado de

https://conrado.ucf.edu.cu/index.php/c onrado/article/view/1158/1164

Barrios, S., Rubio, M., Gutiérrez, M., y Sepúlveda, C. (2012). Aprendizajeservicio como metodología para el desarrollo del pensamiento crítico en Educación Superior. Revista Cubana de Educación Médica Superior, 26(4), 594603.

Batlle, R. (2013). El AprendizajeServicio en España: el contagio de una revolución pedagógica necesaria. Madrid, España: PPC.

Berríos, V., Contreras, M., Herrada, M., Robles, M., y Rubio, X. (2012).

Resultados de Aprendizaje Servicio en la UC: Informe final de investigación.

Santiago: Programa Aprendizaje Servicio, Pontificia Universidad Católica de Chile.

Caiceo, J. (2012). Pensamiento pedagógico en Chile en el siglo XX: Desde la escuela nueva al constructivismo. Teoria E Prática Da Educação, 14(2), 7-20.

doi: 10.4025/tpe.v14i2.16148

Caire, M., González, M., Jouannet, C., Montalva, J., y Ponce, C. (2018). Guía para la Institucionalización de Aprendizaje Servicio de una carrera.
Centro de Desarrollo Docente. Pontificia Universidad Católica de Chile.

Recuperado de

https://cld.bz/skUYaPu/74/

Colén, M., y Castro, L. (2017). El desarrollo de la relación teoría y práctica en el grado de maestro en educación primaria. Profesorado. Revista de currículum y formación de profesorado, 21(1), 59-79.

Curry, B. (1992). Instituting Enduring Innovations: Achieving Continuity of Change in Higher Education. ASHEERIC Higher Education Report, 7.

Furco, A. (1999). Self-Assessment Rubric for the Institutionalization of Service-Learning in Higher Education. Recuperado de https://digitalcommons.unomaha.edu/c gi/viewcontent.cgi?article $=1105 \&$ contex $\underline{\mathrm{t}=\mathrm{slces} \text { lgen }}$

Furco, A. (2001). Advancing

Service-Learning at Research Universities. New Directions for Higher Education, 114, 67-78.

Furco, A., y Billig, S. (2002). ServiceLearning: The Essence of Pedagogy. Connecticut: Information Age Publishing.

Galicia, L., Balderrama, J., y Edel, R. (2017). Validez de contenido por juicio de expertos: propuesta de una herramienta virtual. Apertura, 9(2), 4253. doi:10.32870/Ap.v9n2.993

García, M., y Cotrina, M. (2015). El aprendizaje y servicio en la formación inicial del profesorado: de las prácticas educativas críticas a la 
institucionalización curricular.

Profesorado. Revista de Currículum y

Formación de Profesorado, 19(1), 8-25.

Gil, R. (2018). La formación docente:

Horizontes y rutas de innovación.

Ciudad Autónoma de Buenos Aires,

Argentina: CLACSO.

Gil-Gómez, J., Moliner-García, O., Chiva-Bartoll, O., y García, R. (2014).

Una experiencia de Aprendizaje-

Servicio en futuros docentes: desarrollo de la competencia social y

ciudadna. Revista complutense de educación, 27(1), 53-73.

González, M., Jouannet, C., y González, T. (2016). Metodología aprendizaje servicio $(A+S)$ : Una oportunidad de desarrollo de habilidades transversales en estudiantes universitarios. Educacion y diversidad = Education and diversity: Revista inter-universitaria de investigación sobre discapacidad e interculturalidad, 10(2), 115-126.

Groh, C., Stallwood, L., y Daniels, J. (2011). Service-learning in nursing education: Its impact on leadership and social justice. Nursing education perspectives, 32(6), 400-405.

Gutiérrez-Torres, A., y BuitragoVelandia, S. (2019). Las Habilidades Socioemocionales de los Docentes, herramientas de paz en la escuela. Praxis \& Saber, 10(24), 167-192. doi:10.19053/22160159.v10.n25.2019. 9819

Jenkins, A. y Sheehey, P. (2011). A checklist for implementing ServiceLearning in higher education. Journal of Community Engagement and
Scholarship, 4(2), 52-60.

Jouannet, C. (Junio de 2013). Impactos del Aprendizaje Servicio en profesorado, socios comunitarios y alumnado: El modelo de la Universidad Católica de Chile. En UniversidadComunidad: Creando sinergias. Ponencia llevada a cabo en Congreso Nacional de Aprendizaje-Servicio Universitario ApS(U), Red Universitaria Española de Aprendizaje-Servicio (ApSU), Madrid, España.

Jouannet, C., Ponce, C., y Contreras, A. (2012). Impacto de la metodología Aprendizaje-Servicio $(A+S)$ según la percepción de los docentes. En M. A. Herrero y M. N. Tapia (Comp.), Actas de la II Jornada de Investigadores sobre Aprendizaje-Servicio (pp. 65-68). Buenos Aires, Argentina: CLAYSS-Red Iberoamericana de aprendizaje-servicio.

Jouannet, C., Salas, M., y Contreras, M. (2013). Modelo de implementación de aprendizaje servicio $(A+S)$ en la UC. Una experiencia que impacta positivamente en la formación profesional integral. Calidad en la educación, 39, 198-212.

Jouannet, C., Montalva, J., Ponce, C., y Von Borries, V. (2015). Diseño de un modelo de institucionalización de la metodología de aprendizaje servicio en educación superior. RIDAS, Revista Iberoamericana de Aprendizaje y Servicio, 1, 112-131. doi:10.1344/RIDAS2015.1.7

Mayor, D., y Rodríguez, D. (2016). Aprendizaje-servicio y práctica docente: Una relación para el cambio educativo. Revista de Investigación Educativa, 
$34(2), 535-552$.

doi: $10.6018 /$ rie.34.2.231401

Menor, M., Aguilar, M., Mur, N., y Santana, C. (2017). Efectividad de las intervenciones educativas para la atención de la salud. Revisión sistemática. MediSur, 15(1), 71-84.

Otzen, T., y Manterola, C. (2017). Técnicas de muestreo sobre una población a estudio. International Journal of Morphology, 35(1), 227-232. doi: $10.4067 /$ S071795022017000100037

Páez, M., y Puig, J. M. (2013). La reflexión en el Aprendizaje-Servicio. Revista Internacional de Educación para la Justicia Social (RIEJS), 2(2), 13-32.

Pizarro, V., y Hasbún, B. (2019). Aprendizaje servicio en la educación superior chilena. Recuperado de https://libros.uchile.cl/953

Quilaqueo D., y San Martín, D. (2008). Categorización de saberes educativos mapuche mediante la teoría fundamentada. Estudios Pedagógicos, 34(2), 151-168.

Salum, J. (2018). Aprender a desaprender para un aprendizaje transformativo: Una mirada epistemológica. Revista Observatório, 4(5), 900-922. doi:10.20873/uft.24474266.2018v4n5p900

Sandia, B., y Montilva, J. (2020). Tecnologías Digitales en el AprendizajeServicio para la Formación Ciudadana del Nuevo Milenio. RIED. Revista Iberoamericana de Educación a Distancia, 23(1), 129-148. doi: $10.5944 /$ ried.23.1.24138

Santamaría-Goicuria, I., y Martínez, A. (2018). El aprendizaje servicio, interculturalidad y justicia social: Experiencias disruptivas y transformadoras con futuras maestras de educación infantil y primaria. Revista Qurriculum, 31, 97-118.

doi:10.25145/j.qurricul.2018.31.005

Schön, D. (1998). El profesional reflexivo. Cómo piensan los profesionales cuando actúan. Barcelona, España: Paidós.

Seid, G. (Noviembre de 2016). Procedimientos para el análisis cualitativo de entrevistas. Una propuesta didáctica. En Métodos, metodologías y nuevas epistemologías en las ciencias sociales: desafíos para el conocimiento profundo de Nuestra América. Ponencia llevada a cabo en $\mathrm{V}$ Encuentro Latinoamericano de Metodología de las Ciencias Sociales (ELMeCS), Centro Interdisciplinario de Metodología de las Ciencias Sociales Universidad Nacional de La Plata, Mendoza, Argentina.

Sigmon, R. (1979). Service-learning: Three Principles. Synergist, 8(1), 9-1.

Solís, M., Núñez, C., Contreras, I., Vásquez, N., y Ritterhaussen, S. (2016). Inserción profesional docente: Problemas y éxitos de los profesores principiantes. Estudios pedagógicos, $42(2), 331-342$.

Sotelino, A., Mella, I., y RodríguezFernández, J. (2019). Aprendizajeservicio y desarrollo de la expresión corporal en la formación del 
profesorado de educación infantil.

Publicaciones, 49(4), 199-218.

doi: $10.30827 /$ publicaciones.v49i4.1173

6

Strauss, A., y Corbin, J. (2002). Bases

de la investigación cualitativa.

Colombia: Universidad de Antioquia.

Zayas, B., Gozálvez, V., y Gracia, J.

(2019). La Dimensión Ética y

Ciudadana del Aprendizaje Servicio:

Una apuesta por su institucionalización

en la Educación Superior. Revista

Complutense de Educación, 30(1), 1-

15. doi: 10.5209/RCED.55443 